\title{
Nanotechnology-based approach for safer enrichment of semen with best spermatozoa
}

\author{
Casey L. Durfey ${ }^{1}$, Sabrina E. Swistek ${ }^{1,2}$, Shengfa F. Liao ${ }^{1}$, Mark A. Crenshaw ${ }^{1}$, Henry J. Clemente ${ }^{3}$,
} Rooban V. K. G. Thirumalai ${ }^{4}$, Christy S. Steadman ${ }^{1}$, Peter L. Ryan ${ }^{1,5}$, Scott T. Willard ${ }^{1,2}$ and Jean M. Feugang ${ }^{1 *}$ (i)

\begin{abstract}
Background: Advances in nanotechnology have permitted molecular-based targeting of cells through safe and biocompatible magnetic nanoparticles (MNP). Their use to detect and remove damaged spermatozoa from semen doses could be of great interest. Here, MNP were synthesized and tested for their ability to target apoptotic (annexin V) and acrosome-reacted (lectin) boar spermatozoa, for high-throughout retrieval in a magnetic field (nanoselection). The potential impacts of nanoselection on sperm functions and performance of offspring sired by sperm subjected to nanoselection were determined. Fresh harvested and extended boar semen was mixed with various amounts $(0,87.5$, and $175 \mu \mathrm{g})$ of MNP-conjugates (Annexin V-MNP or Lectin-MNP) and incubated (10 to $15 \mathrm{~min}$ ) for $37^{\circ} \mathrm{C}$ in Exp. 1. In Exp. 2, extended semen was mixed with optimal concentrations of MNP-conjugates and incubated (0, 30, 90, or $120 \mathrm{~min})$. In Exp. 3, the synergistic effects of both MNP-conjugates ( $87.5 \mu \mathrm{g}-30 \mathrm{~min})$ on spermatozoa was evaluated, followed by sperm fertility assessments through pregnancy of inseminated gilts and performance of neonatal offspring. Sperm motion, viability, and morphology characteristics were evaluated in all experiments.
\end{abstract}

Results: Transmission electron microscopy, atomic force microscopy, and hyperspectral imaging techniques were used to confirm attachment of MNP-conjugates to damaged spermatozoa. The motility of nanoselected spermatozoa was improved $(P<0.05)$. The viability of boar sperm, as assessed by the abundance of reactive oxygen species and the integrity of the acrosome, plasma membrane, and mitochondrial membrane was not different between nanoselected and control spermatozoa. The fertility of gilts inseminated with control or nanoselected spermatozoa, as well as growth and health of their offspring were not different between $(P>0.05)$.

Conclusions: The findings revealed the benefit of magnetic nanoselection for high-throughput targeting of damaged sperm, for removal and rapid and effortless enrichment of semen doses with highly motile, viable, and fertile spermatozoa. Therefore, magnetic nanoselection for removal of abnormal spermatozoa from semen is a promising tool for improving fertility of males, particularly during periods, such as heat stress during the summer months.

Keywords: Acrosome reaction, Apoptosis, Artificial insemination, Boar, Iron oxide nanoparticles, Nanopurification, Nanoselection, Nanotechnology, Reproduction, Swine

\footnotetext{
* Correspondence: jn181@ads.msstate.edu

${ }^{1}$ Department of Animal and Dairy Sciences, Mississippi State University,

Mississippi State, MS, USA

Full list of author information is available at the end of the article
}

(c) The Author(s). 2019 Open Access This article is distributed under the terms of the Creative Commons Attribution 4.0 International License (http://creativecommons.org/licenses/by/4.0/), which permits unrestricted use, distribution, and reproduction in any medium, provided you give appropriate credit to the original author(s) and the source, provide a link to the Creative Commons license, and indicate if changes were made. The Creative Commons Public Domain Dedication waiver (http://creativecommons.org/publicdomain/zero/1.0/) applies to the data made available in this article, unless otherwise stated. 


\section{Background}

Numerous factors associated with the boar (e.g., genetic, health, nutrition, etc.), the environment (e.g., seasonal variations, stress, etc.), and the post-collection semen manipulation (e.g., cryopreservation, etc.) are known to affect sperm quality and fertility potential $[1,2]$. Consequently, the causes of poor semen quality are multifactorial $[3,4]$, and reactive oxygen species (ROS), excreted by non-viable spermatozoa within the semen, are harmful to intact spermatozoa $[5,6]$. The removal of non-viable or damaged spermatozoa from semen doses is essential to maintain high reproductive performance of males. To this end, the current progress in nanotechnology gives new prospects to develop novel non-destructive and non-invasive techniques for sperm manipulation in livestock $[7,8]$.

Nanotechnology is a new field of science dealing with molecules less than $100 \mathrm{~nm}$ in diameter, known as nanoparticles. Their unique physico-chemical properties and tunable synthesis make them suitable tools for various bio-applications [7, 9], with promising potentials in reproductive sciences [10-14]. The use of nanoparticles to target physical and physiological characteristics of sperm (motility, directionaliy, apoptosis, intact acrosome, etc.) can help predict whether a semen sample is suitable for assisted reproductive techniques (ART), leading to successful fertilization $[15,16]$. Routine techniques for sperm purification such as swim-up [17], discontinuous percoll [18], albumin filtration [19], density gradient centrifugations (DGC) [8], and magnetic-assisted cell sorting (MACS) $[20,21]$ yield low numbers of motile spermatozoa, but appear more suitable for small-scale applications such as in vitro fertilization (IVF) and intra-cytoplasmic sperm injection (ICSI). Comparative studies have revealed the preponderant effects of MACS for the selection of viable spermatozoa $[16,20]$, leading to better reproductive outcomes than other techniques (i.e., DGC). Despite the numerous advantages of MACS (e.g., simple, rapid, affordable), its performance is limited to less than $10^{9}$ spermatozoa processed for a single target of sperm viability parameter (i.e., apoptosis) [16, 22], which drastically limits its applicability in the swine industry.

Therefore, the use of silane- and polyvinylpyrrolidonecoated colloid silica in density (DLC) and single (SLC) layer centrifugation protocols has revealed beneficial for selecting high quality spermatozoa [23-26], through the purification of high sperm number per analysis (i.e., up to $100 \times 10^{6}$ boar spermatozoa). However, the molecular mechanism of the SLC technique remains unclair [23], and its cost and low recovery yield may limit routine applications in swine farms [24, 27]. Recent studies have reported the use of conjugated magnetic nanoparticles as novel tools for molecular-based selection of spermatozoa regardless of the species [28-30]. These later studies used iron oxide nanoparticle conjugated with annexin V, to target early apoptotic spermatozoa through the externalized phosphatidylserine residues on their surface membrane and lectin, to bind carbohydrates on prematurely acrosome-reacted spermatozoa [22, 29, 31]. Indeed, both apoptosis and acrosome reaction are common defects that characterize non-viable spermatozoa due to increased ROS levels influencing cellular metabolism [32, 33]. The simultaneous targeting of both sperm defects for removal from semen doses would be of great interest in swine farms.

Despite these efforts, swine producers are still unwilling to accept these technologies, and research should continue toward the most acceptable and friendly protocol for substantial reduction of productivity losses in swine farms $[3,34]$. Here we used magnetic iron oxide nanoparticles (MNP) that were conjugated to annexin $\mathrm{V}$ or lectin to allow a two-step procedure removal of both apoptotic and acrosome-reacted spermatozoa from boar semen doses. Synthesized MNP conjugates were characterized and their effectiveness to interact with boar spermatozoa was confirmed in various exposure conditions (dose-dependent effects and time-dependent incubations). The preservation of sperm function following nanoselection was evaluated both in vitro, through sperm motion and viability characteristics and in vivo, through field fertility trial and neonatal offspring performance.

\section{Methods}

Reagents, nanoparticle synthesis, semen and animal care Iron oxide or magnetic nanoparticles (MNP) were synthesized and coated by Clemente Associates (Madison, CT, USA) following an undisclosed proprietary protocol. Coating with lectins (PNA/ LCA) or annexin V (Sigma Aldrich; St Louis, MO, USA) allowed MNP to selectively bind with glycans exposed by damaged acrosomal membranes or early apoptotic spermatozoa, respectively. Stock solutions $\left(1 \mathrm{mg} / \mathrm{mL}=\sim 2.5 \times 10^{6}\right.$ particles $\left./ \mathrm{mg}\right)$ of each Annexin V-MNP and Lectin-MNP, containing sodium azide were stored at $4{ }^{\circ} \mathrm{C}$ until use. Insemination doses $\left(\sim 3-4 \times 10^{9}\right.$ spermatozoa/80 mL) of freshly extended boar semen (Yorkshire $\times$ Duroc) were purchased (Prestage Farms; West Point, MS, USA) for experiments. Purchased mature crossbred gilts (Yorkshire $\times$ Duroc; Prestage Farms) were maintained at the Leveck Animal Research Center (Mississippi Agricultural and Forestry Experiment Station, Mississippi State University) and fed with ad libitum access to water. Animal handling was performed according to protocols approved by the Institutional Animal Care and Use Committee of Mississippi State University. All other reagents were purchased from Sigma-Aldrich. 


\section{Characterization of synthesized MNP conjugates}

Aliquots of each synthesized Lectin-MNP and Annexin V-MNP conjugates were placed on transmission electron microscope (TEM) grids and allowed to dry for at least one day. Thereafter, loaded grids were submitted to imaging (TEM-JEOL 2100, $200 \mathrm{kV}$; instrument with Gatan Orius 832 camera).

\section{Exp. 1: Dose-dependent targeting of spermatozoa and nanoselection \\ Dose-dependent targeting}

Various amounts of each MNP conjugate $(0,87.5$, or $175 \mu \mathrm{g}$ ), corresponding to $0,87.5$, or $175 \mu \mathrm{L}$ of stocks were tested to determine the optimal amount of each MNP conjugate necessary to achieve effective nanoselection. These volumes corresponded to $0, \sim 5500$ and $\sim 11,000$ particles $/ \mathrm{mL}$ of spermatozoa $\left(40-50 \times 10^{6} / \mathrm{mL}\right)$.

\section{Nanoselection procedure}

An illustration of the sperm nanoselection process is shown in Fig. 1. This process consisted to the specific targeting and removal of apoptotic or acrosome-reacted spermatozoa, using Annexin V- or Lectin-conjugated MNP, respectively. For each individual MNP conjugate, freshly harvested and extended boar semen samples
(40 mL) were mixed with Annexin V- or Lectin-conjugated MNP (Fig. 1a), incubated for $10-15$ min at $37^{\circ} \mathrm{C}$ with a gentle rotation, and placed against a strong (12,000 gauss) neodymium magnet, for $10 \mathrm{~min}$ at room temperature, to entrap free MNP conjugates and sperm-MNP complexes (Fig. 1b). Unbound or nanoselected (non-apoptotic or acrosome intact) spermatozoa were eluted into new identified tubes (Fig. 1c) for motility and viability analyses. The experiment was repeated at least four times for each MNP conjugate, using single boar semen doses $(71.2 \% \pm 1.1 \%$ total motility).

\section{Exp. 2: Incubation time for optimal sperm labeling and nanoselection}

Freshly harvested and extended boar semen were incubated with the optimal amount of each MNP conjugate (from Exp.1) and incubated 0, 30, 90, or $120 \mathrm{~min}$ at $37^{\circ}$ C. All procedures were performed as described in Exp. 1 , followed by motility, viability, and morphology analyses to determine the optimal co-incubation time. The experiment was repeated at least four times for each MNP conjugate, using single boar semen doses $(72.0 \% \pm$ $0.6 \%$ total motility).

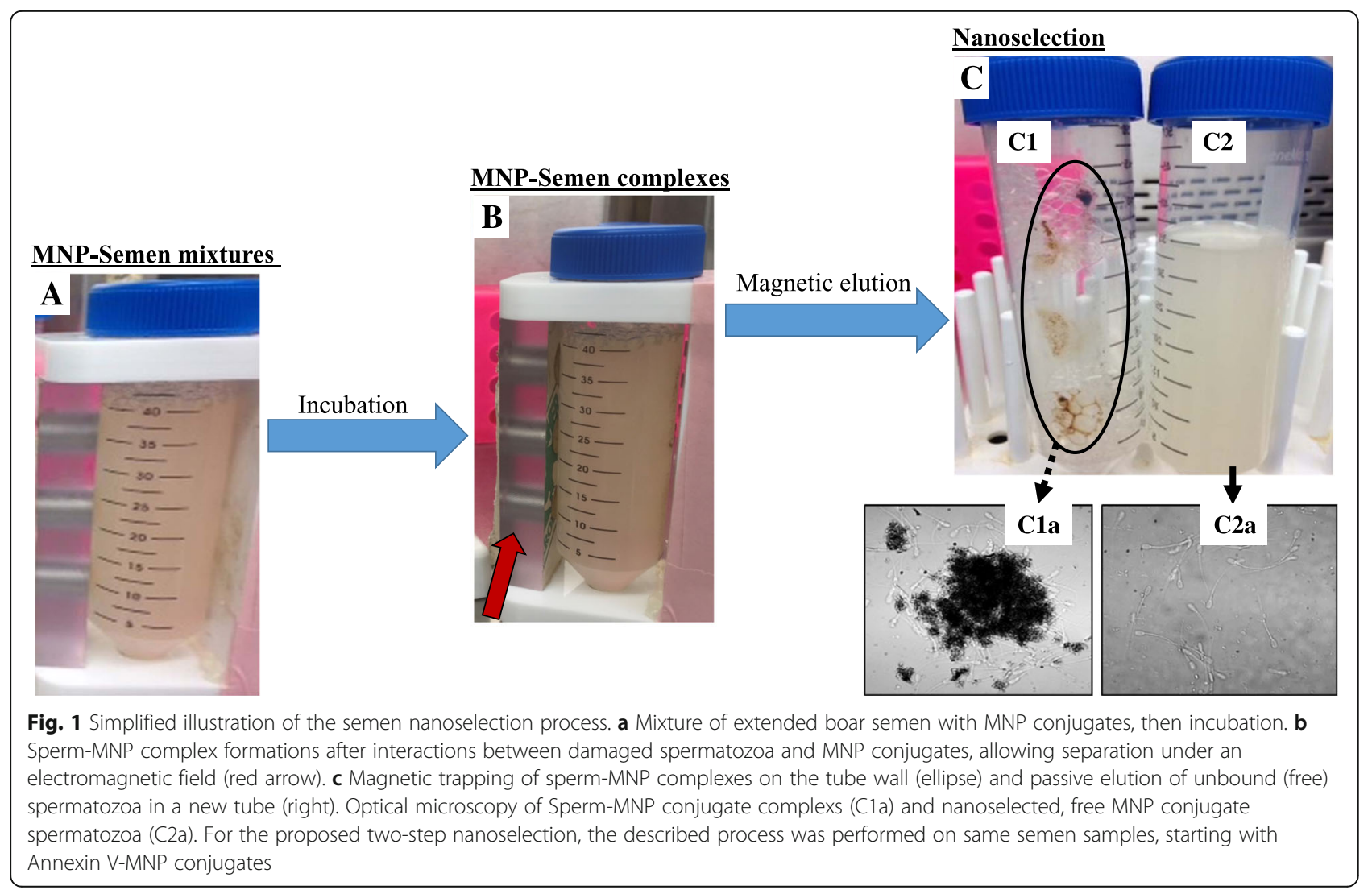


Exp. 3: Evaluation of the two-step sperm targeting and nanoselection

\section{Two-step targeting for nanoselection}

The 1-step procedure corresponded to the removal of apoptotic spermatozoa from extended fresh semen $(40 \mathrm{~mL})$ that were mixed with the optimal amount $(87.5 \mu \mathrm{g})$ of Annexin V-conjugated MNP and incubated for the optimal time $(30 \mathrm{~min})$. Thereafter, the mixtures were submitted to nanoselection, as shown in Fig. 1. Resulting nanoselected (non-apoptotic) spermatozoa were eluted into new identified tubes (1-step), and immediately mixed with the optimal amount of Lectin-conjugated MNP for the optimal incubation time to target and remove acrosome reacted spermatozoa. Thereafter, nanoselected spermatozoa (non-apoptotic and acrosome intact) were eluted (2-step) and aliquots were used for various analyses.

\section{Maintenance assessment of sperm function}

Nanoselected spermatozoa obtained from the two-step procedure, corresponding to 1-step followed by the 2-step were used for various analyses (i.e., motility, viability, and morphology characteristics). In addition, the maintenance of the fertility potential of nanoselected spermatozoa originated from the 2 -step procedure was tested.

\section{Evaluation of sperm-MNP interactions}

Aliquots of fresh control or non-selected, MNP-bound (Fig. 1b), and nanoselected (Fig. 1c) spermatozoa were constituted during nanoselection with each MNP conjugate. All samples were subjected to standard preparations for transmission electron (TEM) and atomic/ magnetic force (AFM/MFM) microscopes, and hyperspectral imaging (HI) as previously reported [35, 36]. Briefly, samples were fixed 1) and stained with or without lead and uranyl acetate for TEM-JEOL, 2) in 4\% paraformaldehyde and smeared on histology microscope slides for AFM/MFM, and 3) on histology glass slides with hyperspectral data collected using reference spectral libraries created by MNP-conjugates (CytoViva ${ }^{\circ}$ imaging technology; CytoViva Inc., Auburn, AL, USA).

Analysis of sperm motion and morphology characteristics Analyses were performed using the HTM-IVOS (Hamilton-Thorne Biosciences; Beverly, MA, USA) or CEROS II (IMV Technologies; Maple grove, MN, USA) Computer-Assisted Sperm Analyzers. Aliquots of nanoselected spermatozoa were submitted to analyses with pre-set values of CASA, as previously described [29]. Each sample aliquot was run in triplicate (3 chambers) with an average of $262 \pm 3$ spermatozoa analyzed per chamber. Sperm motility [percent of total, progressive, rapid $(\geq 30 \mu \mathrm{m} / \mathrm{s})$ motility, and static], velocity $[\mu \mathrm{m} / \mathrm{s}$; average path or VAP, straight line or VSL, and curvilinear or $\mathrm{VCL}$ ], and directionality [lateral head amplitude (ALH, $\mu \mathrm{m})$, beat cross frequency $(\mathrm{BCF}, \mathrm{Hz})$, straightness (STR; VSL/VAP $\times 100)$, linearity (LIN; VSL/VCL $\times 100)]$ parameters were evaluated. The proportions of morphologically abnormal spermatozoa, such as bent tail (BT), coiled tail (CT), and distal (DD) and proximal (PD) cytoplasmic droplets were also recorded using CEROS II.

\section{Analysis of sperm viability}

Staining protocols adapted from Feugang et al. [37] and Martinez-Alborcia et al. were used [38]. Nanoselected spermatozoa were diluted to $30 \times 10^{6}$ cells $/ \mathrm{mL}$ with a pre-warmed phosphate-buffered saline solution (PBS). Aliquots of $0.1 \mathrm{~mL}$ of sperm suspensions were allocated to various staining for viability assessments: $2 \mu \mathrm{L}$ propidium iodide (PI, $1 \mathrm{mg} / \mathrm{mL}$ in PBS) for plasma membrane integrity, $5 \mu \mathrm{L}$ PNA-FITC $(100 \mathrm{mg} / \mathrm{mL}$ in PBS) for acrosomal reaction, $2 \mu \mathrm{L}$ JC-1 $(500 \mathrm{mg} / \mathrm{mL}$; Cayman Chemical Co.; Ann Arbor, MI, USA) for mitochondrial integrity, and $2.5 \mu \mathrm{L} \mathrm{H}_{2}$-DCFDA ( $1 \mathrm{mmol} / \mathrm{L}$ in DMSO) for reactive oxygen species (ROS) accumulation within the cells. All samples were incubated at $37^{\circ} \mathrm{C}$ for $15 \mathrm{~min}$, then diluted six times with prewarmed-PBS to reduce both dye and sperm concentrations for adequate and immediate analyses with flow cytometry (BectonDickinson FACSDiva version 6.1.3). A total of 10,000 events were set to evaluate the proportions of stained spermatozoa (control and nanoselected) and their relative fluorescence intensities to assess sperm damage. Sample aliquots were mounted onto individual microscope slides to confirm the successful staining under an epifluorescence microscope (EVOS FL-Auto, Thermo Fisher Scientific; Hampton, NH, USA).

\section{Field fertility test and offspring performance}

The fertility maintenance of nanoselected spermatozoa was tested as an indispensable step prior to any large-scale application. Estrus was detected on sexually mature gilts $(\sim 8$ months old) through responsiveness to a teaser boar or a lordosis test ("standing reflex"). Each responding gilt was inseminated twice within $24 \mathrm{~h}$, starting from $6 \mathrm{~h}$ post-estrus detection, with single sire doses (two total) of either control (non-selected; $n=7$ gilts) or nanoselected ( $n=7$ gilts) semen. All inseminated gilts $(n=14)$ were housed in a partially open building during breeding and gestation. Pregnant gilts were later transported to a fully enclosed building for farrowing and lactation within crates. The numbers of pregnant gilts, weaned pigs, and litter sizes were recorded.

Neonatal pigs (1-2 per litter/sperm group) were randomly selected for umbilical blood collection at birth and before nursing. Blood samples of eight neonatal pigs born from each control or nanoselected spermatozoa 
were harvested into EDTA-coated tubes. Whole blood aliquots were taken for glucose (Glucose meter, Agamatrix Inc., Salem, NH) and pact cell volume or hematocrit analysis, using standard procedures, and the remaining whole blood samples were centrifuged $(1500 \mathrm{r} / \mathrm{min}$ for $15 \mathrm{~min}$ at $4{ }^{\circ} \mathrm{C}$ ) and blood plasma were collected and stored at $-80^{\circ} \mathrm{C}$ for immunoglobulin $\mathrm{G}$ analyses (porcine IgG ELISA kit; Bethyl Laboratories, Inc; Montgomery, TX, USA).

The morphometric parameters of all neonate pigs $(n=$ 83) were taken weekly, from birth until weaning ( $\sim 28 \mathrm{~d}$ post-natal). Weight, growth, crown-rump length (CRL, distance from crown of the head to the base of the tail), body length (distance from tip of snout to base of tail), head length (distance from tip of snout to base of neck), head circumference, and heart girth were measured.

\section{Statistical analysis}

All statistical analysis was performed using the Statistical Analysis Software (SAS) 9.4 (SAS Institute, Inc., Cary, $\mathrm{NC}$ ). A frequency model was used to determine pregnancy rates, and evaluate fecundity characteristics. A general linear model was used to determine differences between and within control and nanopurified semen in all experiments. A linear mixed model was used to evaluate offspring biochemical analyses from neonatal pigs, with two-way interactions as fixed effects including treatment, and gender. Additionally, a linear mixed model was used to evaluate offspring morphometric analyses from birth until weaning from all neonatal pigs, with treatment, gender, and two-way (treatment $\times$ gender and treatment $\times$ day) interactions as fixed effects. Repeated measures of morphometric analyses were analyzed using an autoregressive one covariance method. Litter within trial was considered a random effect when applicable. All data are expressed as mean \pm standard error mean (SEM), with significant differences set as $P \leq 0.05$.

\section{Results}

\section{Characterization of synthesized MNP conjugates}

Results are shown in Fig. 2. The TEM imaging revealed the spherical shape of the synthesized MNP nanoparticles in Fig. 2a, while high resolution (HR) TEM imaging of MNP cores showed planes with interplanar distances (or d-spacing) of $2.53 \AA$ and $2.98 \AA$ (insert in picture B) for Annexin V- and Lectin-MNP, respectively (Fig. 2b and c). Images of both MNP conjugates indicated core diameters of approximately $7.1 \pm 0.2 \mathrm{~nm}$ and $14 \pm 0.4 \mathrm{~nm}$, respectively.

\section{Evaluation of sperm-MNP interactions}

Representative TEM (A), AFM (B), and Hyperspectral (C) imaging are shown in Fig. 3. The TEM imaging

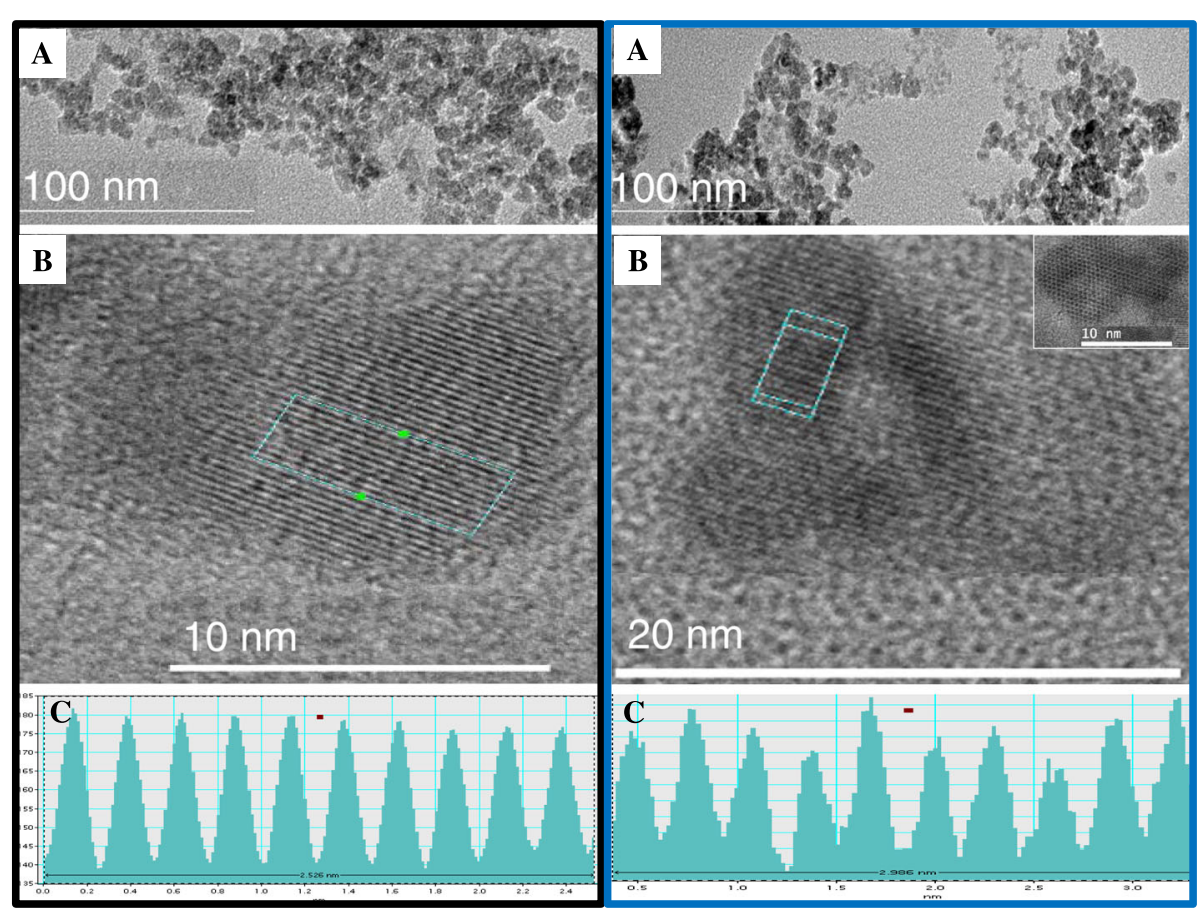

Fig. 2 Standard (A) and high resolution (B) transmission electron microscope of Lectin (Black box)- and Annexin V (Blue box-conjugated MNP. The MNP cores were measured at $7.1 \pm 0.2$ and $14 \pm 0.2 \mathrm{~nm}$ (mean \pm SEM) in diameters, corresponding to Lectin-MNP and Annexin V-MNP, respectively. The insert in micrograph $B$ shows a lattice interplanar distance or $\mathrm{d}$-spacing of the MNP core. Corresponding lattice line intensity graphs $(\mathbf{C})$ revealed average d-spacings of $2.98 \AA$ (hematite or a- $\mathrm{Fe}_{2} \mathrm{O}_{3}$ ), for Lectin-MNP and $2.53 \AA$ (maghemite or $\mathrm{\gamma}$ - $\mathrm{Fe}_{2} \mathrm{O}_{3}$ ), for $A n n e x i n ~ V-M N P$ 
revealed single- (A1) and multi-point (A2) attachments of conjugated MNP to sperm membranes. The maintenance of magnetic properties of MNP conjugates interacting with the sperm head, as expected, are seen with AFM/MFM imaging (B). Hyperspectral imaging (C) showed the presence of MNP as red dots, mainly detected within the sperm head region $(\mathrm{C} 3 \mathrm{~b}$, for Annexin V-MNP and C4b, for Lectin-MNP). Control (C2) and nanopurified (C3a and C4a) spermatozoa did not show detectable presence of residual MNP.

\section{Sperm targeting, effectiveness of nanoselection, and sperm motility characteristics \\ Dose-dependent labeling}

Results are summarized in Fig. 4 and Table 1. In this one-step nanoselection process, corresponding to the retrieval of non-apoptotic or acrosome intact, the presence of MNP conjugates was associated with dose-dependent increases in motile $(P<0.05)$ and forward progressive spermatozoa $(P=0.03)$, as well as VAP and VSL $(P<$ $0.05)$ and VCL $(P=0.09)$. These effects contrasted with the decreased proportions of slow and static spermatozoa (Fig. 4 and Table 1). The proportions of sperm with morphological abnormalities (proximal droplets and coiled and bent tails) were dose-dependently decreased by the presence of MNP conjugates (Fig. 5; $P<0.05$ ). Annexin V-MNP conjugates showed similar results and data are therefore not presented. The targeting of 1.6$2.0 \times 10^{9}$ spermatozoa in $40 \mathrm{~mL}$ extender with $87.5 \mu \mathrm{g}$ $(\sim 218,750$ particles appeared as an optimal ratio that was used for the next experiments.

\section{Time-dependent incubation}

Results are shown in Fig. 6 and Table 2. In this one-step nanoselection process, corresponding to the retrieval of non-apoptotic or acrosome intact, all incubation times showed overall benefits on sperm motility characteristics such as the proportions of motile, progressive, and rapid spermatozoa that were significantly increased in comparison to the control (Fig. 6; $P \leq 0.001$ - Table 2; $P=$ 0.02 ). Incubations of $90 \mathrm{~min}$ or lower generally increased the sperm velocity (VAP, VSL, and VSL) and kinematic (ALH, BCF, STR, and LIN) parameters (Table 2; $P<$ 0.0001); however, no (Fig. 6) or moderate (Table 2) effects on various motility characteristics were observed after $120 \mathrm{~min}$ incubation. Incubations with Annexin V-MNP conjugates showed similar results and we choose not to present the data. Overall, the co-incubation of spermatozoa $\left(1.6-2.0 \times 10^{6} / 40 \mathrm{~mL}\right)$ with either MNP conjugate $(87.5 \mu \mathrm{g}, \sim 218,750$ particles $)$ exhibited highest

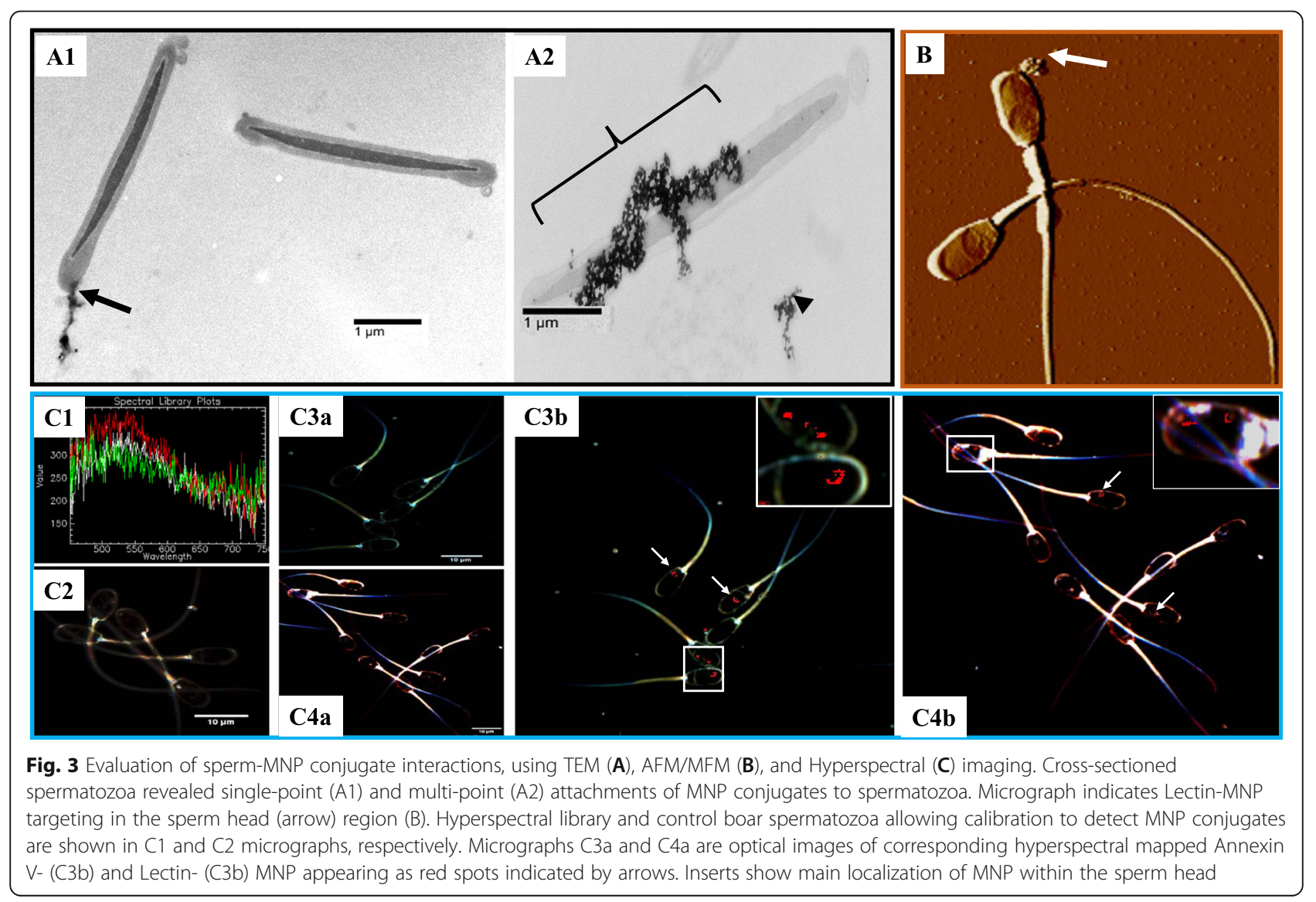




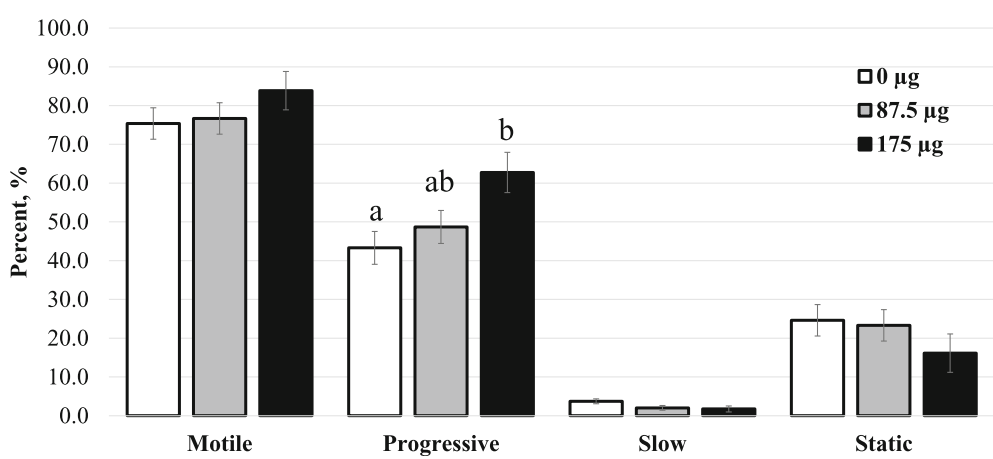

Fig. 4 Motility of nanoselected spermatozoa after dose-dependent labeling/removal procedures. Dose-dependent effects of MNP concentrations were observed. Different letters indicate significant differences between column within the same analyzed parameter $(P=0.034)$. Data are mean \pm sem of at least four independent replicates

effectiveness after $30 \mathrm{~min}$ incubation. This co-incubation time was used for the next experiment.

\section{Two-step targeting}

In this process, the optimal conditions were used for sperm selection, consisting of $30 \mathrm{~min}$ co-incubation of extended semen $\left(1.6-2.0 \times 10^{9}\right.$ spermatozoa $\left./ 40 \mathrm{~mL}\right)$ with $87.5 \mu \mathrm{g}$ of each MNP conjugate. Data presenting both 1-step (non-apoptotic) and 2-step (both non-apoptotic and acrosome intact) nanoselection process are summarized in Fig. 7 and Table 3 . In comparison to the control group, each nanoselection step (1-step or 2-step) significantly increased the proportions of total motile, forward progressive, and rapid spermatozoa, while significantly decreased the proportions of static spermatozoa $(P<$ 0.05 ; Fig. 7a). The first step (1-step, removal of apoptotic) had stronger effects on sperm motility (total and rapid) and velocity parameters (VAP, VSL, and VCL; Fig. 7b) than the second step (2-step; $P<0.05)$. Directionality parameters were variably increased following

Table 1 Dose-dependent effect of MNP- lectin conjugates on sperm motility characteristics

\begin{tabular}{lllll}
\hline $\begin{array}{lllll}\text { Other motion } \\
\text { characteristics }\end{array}$ & \multicolumn{2}{l}{ Nanoparticles $(\mu \mathrm{g})$ per $40 \mathrm{~mL}$ of extended semen } & P-value \\
\cline { 2 - 4 } & $0(\mathrm{~N}=18)$ & $87.5(\mathrm{~N}=19)$ & $175(\mathrm{~N}=15)$ & \\
\hline VAP, $\mu \mathrm{m} / \mathrm{s}$ & $63.9 \pm 4.7^{\mathrm{a}}$ & $77.6 \pm 4.7^{\mathrm{a}}$ & $88.8 \pm 5.8^{\mathrm{b}}$ & 0.04 \\
VSL, $\mu \mathrm{m} / \mathrm{s}$ & $45.4 \pm 2.3^{\mathrm{a}}$ & $49.9 \pm 2.3^{\mathrm{a}}$ & $61.6 \pm 2.8^{\mathrm{b}}$ & $<0.03$ \\
VCL, $\mu \mathrm{m} / \mathrm{s}$ & $128 \pm 11.8$ & $156.6 \pm 11.8$ & $173.5 \pm 14.4$ & 0.09 \\
$\mathrm{ALH}, \mu \mathrm{m}$ & $5.4 \pm 0.6$ & $6.4 \pm 0.6$ & $6.6 \pm 0.7$ & $\mathrm{NS}$ \\
$\mathrm{BCF}, \mathrm{Hz}$ & $38.9 \pm 1.3$ & $38.3 \pm 1.3$ & $36.6 \pm 1.6$ & $\mathrm{NS}$ \\
$\mathrm{STR}, \%$ & $73.9 \pm 3.3$ & $68.4 \pm 3.3$ & $71.9 \pm 4.0$ & $\mathrm{NS}$ \\
LIN, \% & $38.8 \pm 2.7$ & $36.3 \pm 2.7$ & $39.0 \pm 3.3$ & $\mathrm{NS}$ \\
\hline
\end{tabular}

Table represents various sperm kinematic parameters, such as average path velocity (VAP), straight-line velocity (VSL), curve-linear velocity (VCL), head lateral amplitude (ALH), beat flagellum frequency (BCF), straightness (STR VSL/NAP $\times 100)$, linearity $($ LIN - VSL/NCL $\times 100)$. Data are mean $( \pm S E M)$ of $N$ observations of at least 4 independent replicates using pooled semen doses. Different letters on the same line denote significant differences $(P<0.05)$ each removal step $(P<0.05$; Table 3$)$, and the 2-step removal significantly decreased ALH while increasing the straightness (STR) and linearity (LIN) of spermatozoa when compared to control and 1-step counterparts.

\section{Sperm retrieval}

We used the SpermaCue (Minitube USA, Inc., Verona, USA) to evaluate the total sperm counts before and after the two-step targeting process. The results indicated that approximately $3 \%$ of spermatozoa were entrapped ( 96$120 \times 10^{6}$ ) during the two-step targeting, leaving $97 \%$ of free spermatozoa in each insemination dose $(80 \mathrm{~mL})$.

\section{Viability assessment of nanoselected spermatozoa}

Analyses were performed on the 2-step nanoselected spermatozoa (Table 4). The proportions of spermatozoa stained for acrosome reaction (PNA-FITC: $92 \% \pm 7.4 \%$ vs. $98 \% \pm 7.4 \%$ ), plasma membrane integrity (PI: $93 \% \pm 8 \%$ vs. $94 \% \pm 8 \%$ ), mitochondrial potential (JC-1: $86 \% \pm 13 \%$ vs. $88 \% \pm 15 \%)$, and ROS accumulation $\left(\mathrm{H}_{2}\right.$-DCFDA: $99.8 \%$ vs. 99.4\%) were not significantly different between control and nanoselected spermatozoa, respectively $(P>0.05)$. However, relative fluorescence intensities (RFI) associated with ROS production and plasma membrane integrity in nanoselected spermatozoa were lower, but not significantly to the controls $(864 \pm 200$ vs. $1069 \pm 200$ and $9 \pm 26$ vs. $12 \pm 26$, respectively; $P>0.05$ ). Nanoselected spermatozoa showed non-significantly lower JC-1 fluorescence intensity than the control $(898 \pm 224$ vs. $426 \pm 200, P>0.05)$.

\section{Reproductive outcomes of nanoselected spermatozoa}

Pregnancy and farrowing rates resulted for gilts inseminated with control $[43 \%(3 / 7)$ and $100 \%(3 / 3)]$ and nanoselected [57\% (4/7) and 100\% (4/4)] spermatozoa were comparable $(P>0.05)$. Similarly, litter size and weight for gilts inseminated with control $(11.3 \pm 5.6$ and $1.6 \pm$ $0.04 \mathrm{~kg}$, respectively) and nanoselected $(12.7 \pm 7.3$ and 


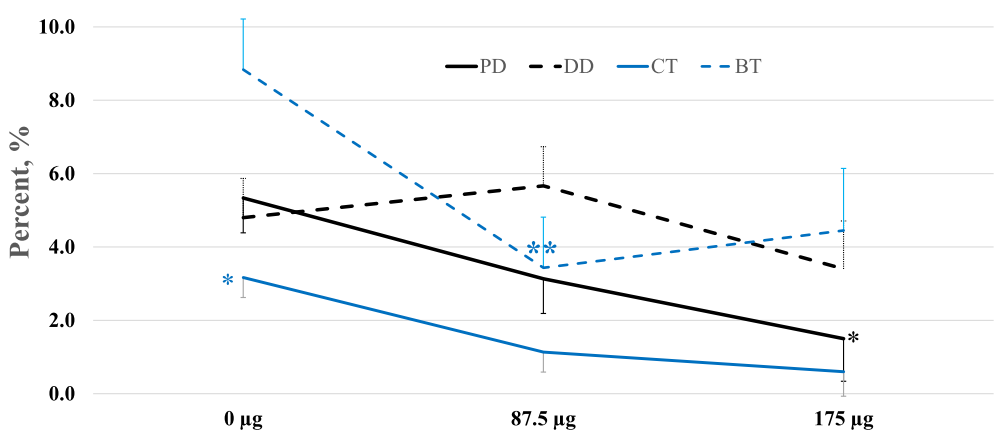

Fig. 5 Morphology of nanoselected spermatozoa after dose-dependent labeling/removal procedures. Asteriks indicate significant differences between MNP concentrations, within the same parameters corresponding to proximal cytoplasmic droplet (PD), distal cytoplasmic droplet (DD), coiled tail $(\mathrm{CT})$, and bent tail $(\mathrm{BT}) .{ }^{*} P<0.05$ indicates difference from other groups and ${ }^{* *} P=0.04$ indicates difference from the control only. Data are mean \pm SEM of at least four independent replicates

$1.5 \pm 0.04 \mathrm{~kg}$, respectively) spermatozoa were comparable $(P>0.1)$, as were the proportion of live pigs weaned in litters of sows inseminated with control (89\%; 40/45) and nanoselected $(92 \% ; 35 / 38)$ spermatozoa.

The glucose $(56 \pm 8$ vs. $48 \pm 8 \mathrm{mg} / \mathrm{dL}$ ), hematocrit $(22 \% \pm 1 \%$ vs. $22 \% \pm 2 \%)$, and IgG (342 \pm 108 vs. $568 \pm$ $112 \mathrm{ng} / \mathrm{mL}$ ) measurements, all indicative of the neonatal pigs health performance, were not significant different between nanoselected- and control-born pigs. Likewise, no significant interactions were found between sperm treatment and offspring gender $(P>0.05)$.

All morphometric parameters showed similar measurements in both control- and nanoselected-born pigs $(P>0.05)$. All born pigs grew at normal and comparable pace, until weaning at $28 \mathrm{~d}$ of age $(8.2 \pm 0.2 \mathrm{~kg}$ vs. $7.7 \pm$ $0.2 \mathrm{~kg}$ for control and nanoselected, respectively). However, the average head length was significantly shorter in nanoselected-born pigs vs. the controls, at the time of weaning $(15.0 \pm 0.2 \mathrm{~cm}$ vs. $16.2 \pm 0.2 \mathrm{~cm}, P<0.01)$, and there were no significant interactions between sperm treatment (control or nanoselected) and the offspring gender $(P>0.1)$.

\section{Discussion}

Semen contain variable proportions of damaged spermatozoa that can be exacerbated during hot seasons, causing substantial productivity losses in commercial studs due to the rejection of poor semen [3, 34]. Numerous in vitro tests are being used to evaluate apoptosis and early acrosome reaction, two main causes of poor semen quality $[11,39,40]$. Obtained results are mainly informative $[11,21]$, and semen not meeting the minimum standards are still rejected. Here we showed that specifically designed magnetic nanoparticle conjugates could be used for smooth, effortless, and high-throughput targeting and removal of damaged spermatozoa from semen doses, without further affecting the functionality and fertility of nanoselected spermatozoa.

In this study, the synthesized iron oxide particles measured 7 to $14 \mathrm{~nm}$ in diameters, and corresponded to

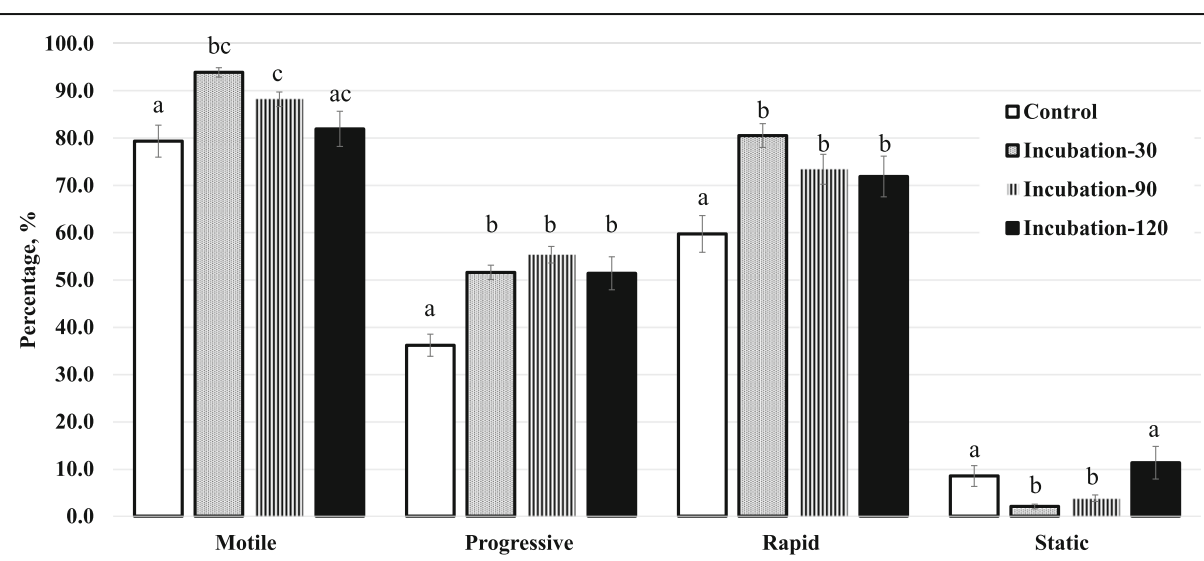

Fig. 6 Motility of nanoselected spermatozoa after dose-dependent incubations. Different letters indicate significant differences between columns (incubation times) of each motility parameter $(P<0.001$ : total motility and progressive, $P=0.001$ : rapid and $P=0.02$ : static). Data are mean \pm SEM of at least four independent replicates 
Table 2 Time-dependent effect of MNP-lectin conjugates on sperm motility characteristics

\begin{tabular}{|c|c|c|c|c|c|}
\hline \multirow{2}{*}{$\begin{array}{l}\text { Other motion } \\
\text { parameters }\end{array}$} & \multicolumn{4}{|c|}{ Incubation time, min } & \multirow[t]{2}{*}{$P$-values } \\
\hline & $\begin{array}{l}0 \\
(\mathrm{~N}=18)\end{array}$ & $\begin{array}{l}30 \\
(\mathrm{~N}=19)\end{array}$ & $\begin{array}{l}90 \\
(\mathrm{~N}=15)\end{array}$ & $\begin{array}{l}120 \\
(\mathrm{~N}=16)\end{array}$ & \\
\hline $\mathrm{VAP}, \mu \mathrm{m} / \mathrm{s}$ & $70 \pm 2^{a}$ & $77.6 \pm 2.1^{b}$ & $73.2 \pm 3.1^{c}$ & $74.8 \pm 1.7^{\mathrm{bc}}$ & $<0.0001$ \\
\hline$V S L, \mu \mathrm{m} / \mathrm{s}$ & $35.4 \pm 1.1^{a}$ & $40.5 \pm 0.8^{b}$ & $45.1 \pm 0.7^{c}$ & $42.7 \pm 1.3^{d}$ & $<0.0001$ \\
\hline$V C L, \mu \mathrm{m} / \mathrm{s}$ & $154.5 \pm 4.1^{\mathrm{a}}$ & $164.7 \pm 4.3^{b}$ & $150.3 \pm 4.8^{\mathrm{a}}$ & $160.4 \pm 2.9^{b}$ & 0.001 \\
\hline $\mathrm{ALH}, \mu \mathrm{m}$ & $8.3 \pm 0.1^{a}$ & $7.7 \pm 0.1^{b}$ & $7.2 \pm 0.3^{c}$ & $7.9 \pm 0.1^{b}$ & $<0.0001$ \\
\hline $\mathrm{BCF}, \mathrm{Hz}$ & $38.1 \pm 0.4^{a}$ & $36.2 \pm 0.5^{b}$ & $37.9 \pm 0.9^{\mathrm{a}}$ & $34.1 \pm 0.6^{c}$ & $<0.0001$ \\
\hline STR, \% & $51.1 \pm 0.6^{\mathrm{a}}$ & $54.2 \pm 0.9^{b}$ & $63.1 \pm 2.5^{c}$ & $58.1 \pm 1.0^{d}$ & $<0.0001$ \\
\hline LIN, \% & $24.3 \pm 0.5^{a}$ & $26.9 \pm 0.5^{b}$ & $33.1 \pm 2.2^{c}$ & $28.2 \pm 0.6^{b}$ & $<0.0001$ \\
\hline
\end{tabular}

The table represents various sperm kinematic parameters, such as average path velocity (VAP), straight-line velocity (VSL), curve-linear velocity (VCL), head lateral amplitude $(A L H)$, beat flagellum frequency $(B C F)$, straightness (STR - VSL/VAP $\times 100)$, linearity (LIN - VSL/VCL $\times 100)$. Data are mean $( \pm$ sem) of N observations of at least 4 independent replicates using pooled semen doses. Different letters on the same line denote significant differences $(P<0.05)$

maghemite $\left(\gamma-\mathrm{Fe}_{2} \mathrm{O}_{3}\right)$ and hematite $\left(\alpha-\mathrm{Fe}_{2} \mathrm{O}_{3}\right)$ as revealed by the HRTEM images showing respective interplanar distances of $2.54 \AA$ and $2.98 \AA$ [41, 42]. All forms of MNP (hematite or $\alpha-\mathrm{Fe}_{2} \mathrm{O}_{3}$, maghemite or $\gamma-\mathrm{Fe}_{2} \mathrm{O}_{3}$, and magnetite or $\mathrm{Fe}_{3} \mathrm{O}_{4}$ ) have been used for in vitro targeting of spermatozoa, in a broad range of applications (i.e., sperm nanopurification, toxicity test, and sperm-mediated gene transfer), without affecting their functionality or structures (reviewed by [43]). In the current study, the conjugation of maghemite-made MNP with annexin V and hematite-made MNP with lectins (PNA/LCA) to respectively target apoptici and acrosome reacted spermatozoa [44-46] did not affect the MNP magnetism nor the functionality of both MNP conjugates. Various imaging technologies (MFM/AFM, TEM and hyperspectral) were used to confirm the presence of MNP conjugates in the sperm head, the expected localization of both defects. Both single- and multi-point attachments of MNP conjugates were observed on spermatozoa, and likely reflected the extent of damages. From this observation, it was speculated that deep damages may attract high number of MNP-conjugates, creating aggregates and necessary differential magnetism force for effective removal of damaged spermatozoa.

Previous studies have used the abovementioned MNP forms to harmessly remove apoptotic spermatozoa from boar and bull semen [29, 31, 47]. Fixed ratios of Lectin-MNP per boar $\left(1 \mathrm{mg} / 33 \times 10^{6}\right)$ [29], and per bull $\left(1 \mathrm{mg} / 10^{6}\right)$ [31] spermatozoa were used, and resulting nanoselected spermatozoa led to full-term pregnancies and birth of healthy offspring. In contrast, the removal of both apoptotic and acrosome reacted spermatozoa was tested in the current study. We first conducted dose-dependent and time-dependent experiments to determine the optimum sperm-to-MNP conjugate combinations, the 2-step protocol. With the expectation to target approximately $20 \%$ of damaged spermatozoa per semen dose $\left(80 \mathrm{~mL}\right.$ containing $3-4 \times 10^{9}$ spermatozoa),
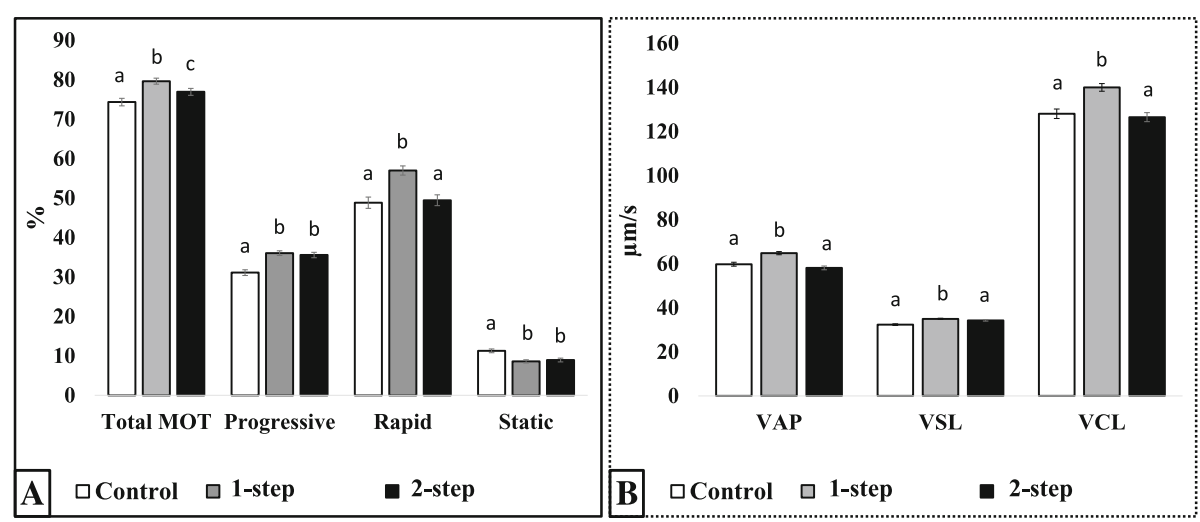

Fig. 7 Motility (A) and velocity (B) characteristics of nanoselected spermatozoa following double or two-step removal. Total motile (total MOT), progressive (forward moving), rapid (fast), and static (dead/non-motile) spermatozoa with their corresponding average path (VAP), straight line (VSL), and curvelinear (VCL) velocities were assessed. Spermatozoa were standard non-selected (control) or successively nanoselected with Annexin-V (1-step) followed by Lectin (2-step) -MNP conjugates. Columns with different letters differ significantly $(P<0.05)$. Data are mean \pm SEM of at least four independent replicates 
Table 3 Additional movement characteristics of double nanoselected boar spermatozoa

\begin{tabular}{lllll}
\hline & ALH, $\mu \mathrm{m}$ & $\mathrm{BCF}, \mathrm{Hz}$ & $\mathrm{STR}, \%$ & $\mathrm{LIN}, \%$ \\
\hline Control & $7.95 \pm 0.08^{\mathrm{a}}$ & $38.14 \pm 0.3^{\mathrm{ab}}$ & $55.7 \pm 0.6^{\mathrm{a}}$ & $27.3 \pm 0.4^{\mathrm{a}}$ \\
1-step removal $^{1}$ & $8.06 \pm 0.05^{\mathrm{a}}$ & $37.6 \pm 0.3^{\mathrm{b}}$ & $55.8 \pm 0.6^{\mathrm{a}}$ & $27.3 \pm 0.4^{\mathrm{a}}$ \\
2-step removal $^{2}$ & $7.5 \pm 0.1^{\mathrm{b}}$ & $38.7 \pm 0.3^{\mathrm{a}}$ & $60.4 \pm 0.8^{\mathrm{b}}$ & $29.8 \pm 0.7^{\mathrm{b}}$
\end{tabular}

${ }^{1}$ Removal of apoptotic spermatozoa (annexin V-MNP conjugates $=1$-step) was followed by those with ${ }^{2}$ acrosome reacted (lectin-MNP conjugates $=2$-step). This consecutive removal process corresponds to the proposed double nanoselection with evaluation of the lateral head amplitude (ALH), beat cross frequency (BCF), straightness (VSL/NAP $\times 100$ ), and linearity (VSL/NCL $\times 100$ ). Data are mean $\left( \pm\right.$ sem) of 4 independent replicates; different superscripts $\left({ }^{a, b}\right)$ within the same column indicate significant differences $(P<0.05)$

we added the equivalent volumes of $87.5 \mu \mathrm{g}(87.5 \mu \mathrm{L})$ and $175 \mu \mathrm{g}(175 \mu \mathrm{L})$ of each MNP conjugate into $40 \mathrm{~mL}$ of extended semen $\left(1.5-2 \times 10^{9}\right.$ spermatozoa). Separate incubations (10-15 min) with $87.5 \mu \mathrm{g}$ of each MNP conjugate had no effects on sperm motility characteristics, but significantly reduced the proportions of abnormal spermatozoa. Using $87.5 \mu \mathrm{g}$ of each MNP conjugate, to avoid MNP toxicity [48, 49], all time-dependent incubations (30 to $120 \mathrm{~min}$ ) were beneficial to sperm motility characteristics (vs. the control). The significantly decreased proportions of static spermatozoa after 30-90 min incubation indicated the ability of MNP conjugates to reduce the percentages of defective spermatozoa within the semen samples. This observation is of great interest as the accepted proportion of damage spermatozoa in boar studs varies from 15\% to $30 \%$ $[50,51]$. Altogether, the optimal condition for successful sperm nanoselection in the current study was summarized as $87.5 \mu \mathrm{g}$ of MNP conjugate (per $40 \mathrm{~mL}$ of extended semen, 1.5-2.0 × 109 spermatozoa), for 30-60 min incubation.

Investigating the efficiency of each MNP conjugate on sperm selection revealed a simultaneous elimination of apoptotic spermatozoa in 1-step, followed by acrosome-reacted spermatozoa in 2-step. This sequential process showed maximum sperm removal during the

Table 4 Viability assessment of nanoselected spermatozoa by Flow cytometry

\begin{tabular}{lll}
\hline & Control $^{1}$ & Nanoselected $^{2}$ \\
\hline Reactive oxygen species & $1,069 \pm 200$ & $864 \pm 200$ \\
Plasma membrane integrity & $11.9 \pm 25.6$ & $8.6 \pm 25.6$ \\
Mitochondrial integrity & $426 \pm 200$ & $898 \pm 224$
\end{tabular}

${ }^{1}$ Non selected (Control) vs. ${ }^{2}$ double-selected (annexin V and lectin; Nanoselected) spermatozoa. Data are relative fluorescence intensities (mean \pm sem) of spermatozoa stained with $\mathrm{H}_{2}$-DCFDA (ROS production), propidium iodide (plasma membrane integrity), or JC-1 (mitochondrial potential). Fluorescence intensity values are proportional to the extent of damages of 4 independent replicates (mean \pm sem). Data within the same line are not significantly different $(P>0.05)$ 1-step, indicating that removed spermatozoa may carry both apoptotic and acrosome damages. Both MNP conjugates (in 2-step) resulted in approximately $3 \%$ of sperm retrieval, corresponding to $96-120 \times 10^{6}$ / insemination dose. This low removal rate may be due to the use of fresh semen harvested from high reproductive boars. Speculating that damaged spermatozoa constitute physical impediments within the semen dose, their successful removal increases the proportion of healthy and viable (nanoselected) spermatozoa, leading to high-quality semen doses available for artificial inseminations. These findings are consistent with the unique work in swine using a one-step nanoselection method with Lectin-MNP [29]. In support of the positive outcomes of the nanoselection process, sperm kinematic parameters such as VSL, STR, and LIN have shown positive correlations with fertilization rates [52], while being used as predictors of effective and smooth sperm displacement within the utero-oviduct tube [53, 54] and male fertility [55].

Altogether, the findings indicated the benefit of the proposed two-step nanoselection, showing complementary and synergy effects of the sequential removal. The 1-step removal (Annexin V-MNP) resulted in improved sperm motility (total, progressive, rapid, and static) and velocity (VAP, VSL, and VCL) parameters, while the 2-step (Lectin-MNP) was advantageous to ALH, BCF, STR, and LIN. Using similar Lectin-MNP, data of 1-step removal were comparable to a previous study with boars [29], which support the proposed sequential process to achieve optimum removal of moribund spermatozoa from semen doses.

Semen enrichment with high-quality nanoselected spermatozoa did not translate into higher viability than their control counterparts. The use of high-quality fresh spermatozoa exhibiting minimal damages may explain this inconsistency. Nanoselected spermatozoa maintained more stable plasma membrane, higher mitochondrial membrane potential, and lower ROS level. These features are vital for effective fertilization [56] and cryotolerance $[57,58]$. A previous study has reported the positive effects of the SLC selection protocol on the freezability of boar spermatozoa and the decreased ROS production post-thaw [38]. These observations prompted us to field fertility trials, which did not reveal significant advantages of the nanoselection in fertility outcomes. The absence of plasmatic biochemical changes between piglets born from control or nanoselected spermatozoa allowed us to rule out any health or toxicity concerns.

\section{Conclusions}

The proposed two-step or sequential nanoselection process allowed for 1) successful molecular-based targeting of apoptotic (Annexin V-MNP) and acrosome-reacted (Lectin-MNP) spermatozoa, 2) effortless, rapid, and 
high-throughput enrichment of semen doses with best spermatozoa, capable to induce normal pregnancy and post-natal development of piglets. The application of this process in studs is likely to decrease semen rejection rates, therefore increasing the potential of using boars with reduced semen quality.

\begin{abstract}
Abbreviations
Å: Angström (=0.1 nm); AFM/MFM: Atomic/Magnetic force microscopy; DLS: Dynamic light scattering; HRTEM: High resolution transmission electron microscope; MNP: Magnetic nanoparticles; MOT: Total motile sperm; PROG: Progressive motile sperm; RAP: Rapid motile spermatozoa; RFI: Relative fluorescence intensities; ROS: Reactive oxygen species; STAT: Static spermatozoa; STR: Straightness spermatozoa; TEM: Transmission electron microscope; VAP: Average path velocity; VCL: Curvelinear velocity; VSL: Straight-line velocity
\end{abstract}

\section{Acknowledgements}

The authors thank Prestage Farms Inc., Mississippi Division for providing semen; Mr. William White and colleagues for assisting in animal and facility management (Leveck Animal Research center, Mississippi State University), Dr. Robert Willis and Ms. Wei Tan at The College of Veterinary Medicine at Mississippi State University for their respective assistance in statistical data analyses and flow cytometry analyses; and Mrs. Amanda Lawrence and Dr. I-Wei Chu at the Institute for Imaging and Analytical Technologies, Mississippi State University. This work was supported by the Office of Research and Economic Development (ORED), College of Agriculture and Life Sciences (CALS)/Mississippi Agriculture, Forestry and Extension Service (MAFES) of Mississippi State University, USDA-NIFA (Hactch project under accession number 359010), and the USDA-ARS Biophotonic Initiative (grant\# 58-6402-3-018)

\section{Funding}

This research was supported by the USDA-ARS Biophotonics (grant \# 58-64023-018), the Undergraduate Research Scholar Program of the College of Agriculture and Life Sciences (CALS) and Mississippi Agricultural and Forestery Experiment Station (MAFES).

\section{Availability of data and materials}

Note applicable.

\section{Authors' contributions}

CLD carried out all experiments and analyzed the data; HC designed and synthesized the MNP conjugates; CD, JMF, and SES conducted sperm nanoselection, imaging analyses (AFM/MFM), motility and viability assessments; RVKGT performed all TEM imaging; CLD, SFL, MAC, CSS, and JMF carried out the animal work; MAC, STW, and PLR assisted in the research design and data interpretation; JMF assisted and supervised all experiments, and wrote the manuscript with CLD. All authors read and approved the final manuscript.

\section{Ethics approval}

The present study was approved by the Institutional Animal care and Use Committee of Mississippi State University (IACUC protocol 14-026).

\section{Consent for publication}

Not applicable.

\section{Competing interests}

The authors declare that they have no competing interests.

\section{Author details}

${ }^{1}$ Department of Animal and Dairy Sciences, Mississippi State University, Mississippi State, MS, USA. ²Department of Biochemistry, Molecular Biology, Entomology and Plant Pathology, Mississippi State, MS, USA. ${ }^{3}$ Clemente Associates, Madison, CT, USA. ${ }^{4}$ Institute of Imaging and Analytic Technology (I2AT), Mississippi State University, Mississippi State, MS, USA. ${ }^{5}$ Department of
Pathobiology and Population Medicine Biochemistry, Mississippi State University, Mississippi State, MS, USA.

Received: 9 July 2018 Accepted: 18 December 2018

Published online: 09 February 2019

\section{References}

1. Bungum M, Humaidan P, Axmon A, Spano M, Bungum L, Erenpreiss J, et al. Sperm DNA integrity assessment in prediction of assisted reproduction technology outcome. Hum Reprod. 2007;22(1):174-9.

2. Lopes S, Sun J-G, Jurisicova A, Meriano J, Casper RF. Sperm deoxyribonucleic acid fragmentation is increased in poor-quality semen samples and correlates with failed fertilization in intracytoplasmic sperm injection. Fertil Steril. 1998;69(3):4.

3. Broekhuijse M, Gaustad A, Bolarin Guillén A, Knol E. Efficient boar semen production and genetic contribution: the impact of low-dose artificial insemination on fertility. Reprod Domest Anim. 2015;50(S2):103-9.

4. Foote $\mathrm{RH}$. Fertility estimation: a review of past experience and future prospects. Anim Reprod Sci. 2003;75(1-2):119-39.

5. Shannon P, Curson B. Toxic Effect and action of dead sperm on diluted bovine semen. J Dairy Sci. 1972;55(5):614-20.

6. Sharma RK, Agarwal A. Role of reactive oxygen species in male infertility. Urology. 1996;48(6):835-50.

7. Feugang JM. Novel agents for sperm purification, sorting, and Imaging. Mol Reprod Dev. 2017;9999:1-10.

8. Said TM, Land JA. Effects of advanced selection methods on sperm quality and ART outcome: a systematic review. Hum Reprod Update. 2011;17(6): 719-33.

9. Jain S, Park SB, Pillai SR, Ryan PL, Willard ST, Feugang JM. Applications of Fluorescent Quantum Dots for Reproductive Medicine and Disease Detection. In: Unraveling the Safety Profile of Nanoscale Particles and Materials - From Biomedical to Environmental Applications. Edited by Gomes AC, Sarria MP. Rijeka: InTech; 2018: Ch. 06.

10. Pankhurst QA, Connolly J, Jones SK, Dobson J. Applications of magnetic nanoparticles in biomedicine. J Phys D Appl Phys. 2003;36(13):R167.

11. Sutovsky P, Kennedy CE. Biomarker-based nanotechnology for the improvement of reproductive performance in beef and dairy cattle. Ind Biotechnol. 2013;9(1):6.

12. Rath D, Tiedemann D, Gamrad L, Johnson L, Klein S, Kues W, et al. Sex-sorted boar sperm-an update on related production methods. Reprod Domest Anim. 2015;50(S2):56-60.

13. Barkalina N, Jones C, Coward K. Nanomedicine and mammalian sperm: lessons from the porcine model. Theriogenology. 2016;85(1):74-82.

14. Feugang JM, Youngblood RC, Greene JM, Willard ST, Ryan PL. Selfilluminating quantum dots for non-invasive bioluminescence imaging of mammalian gametes. J Nanobiotechnol. 2015;13(1):38.

15. Sutovsky P, Lovercamp K. Molecular markers of sperm quality. Reproduction in Domestic Ruminants VII; 2011. p. 247.

16. Hozaien MM, Elqusi KM, Hassanen EM, Hussin AA, Alkhader HA, El Tanbouly SM, et al. A comparison of reproductive outcome using different sperm selection techniques; density gradient, testicular sperm, PICSI, and MACS for ICSI patients with abnormal DNA fragmentation index. Fertil Steril. 2018; 110(4):e19-20

17. Mortimer D. Sperm preparation methods. J Androl. 2000:21(3):357-66.

18. Kaneko S, Oshio S, Kobanawa K, Kobayashi T, Mohri H, lizuka R. Purification of human sperm by a discontinuous Percoll density gradient with an innercolumn. Biol Reprod. 1986;35(4):1059-63.

19. Ericsson RJ, Langevin CN, Nishino M. Isolation of fractions rich in human $Y$ sperm. Nature. 1973;246(5433):421-4.

20. Gil M, Sar-Shalom V, Melendez Sivira Y, Carreras R, Checa MA. Sperm selection using magnetic activated cell sorting (MACS) in assisted reproduction: a systematic review and meta-analysis. J Assist Reprod Genet. 2013:30(4):479-85.

21. Valcarce DG, Herraez MP, Chereguini O, Rodriguez C, Robles V. Selection of nonapoptotic sperm by magnetic-activated cell sorting in Senegalese sole (Solea senegalensis). Theriogenology. 2016;86(5):1195-202.

22. Miltenyi S, Müller W, Weichel W, Radbruch A. High gradient magnetic cell separation with MACS. Cytometry. 1990;11(2):231-8.

23. Morrell J. Colloids: applications in sperm preparation for assisted reproduction. In: Advances in Colloid Science. InTech; 2016. 
24. Morrell JM, Rodriguez-Martinez $\mathrm{H}$. Colloid Centrifugation of Semen: Applications in Assisted Reproduction. American Journal of Analytical Chemistry. 2016;7(08):597.

25. Morrell JM, Rodriguez-Martinez H. Colloid centrifugation of semen: applications in assisted reproduction. Am J Anal Chem. 2016;7(08):597.

26. Morrell J, Lagerqvist A, Humblot $P$, Johannisson A. Effect of Single Layer Centrifugation on reactive oxygen species and sperm mitochondrial membrane potential in cooled stallion semen. Reproduction, Fertility and Development. 2017, 29(5):1039-45.

27. Martinez-Alborcia MJ, Morrell JM, Gil MA, Barranco I, Maside C, Alkmin DV, et al. Suitability and effectiveness of single layer centrifugation using Androcoll-P in the cryopreservation protocol for boar spermatozoa. Anim Reprod Sci. 2013;140(3):173-9.

28. Sutovsky P. New approaches to boar semen evaluation, Processing and Improvement. Reprod Domest Anim. 2015;50(Suppl 2):11-9.

29. Feugang J, Liao S, Crenshaw M, Clemente H, Willard S, Ryan P. Lectinfunctionalized magnetic iron oxide nanoparticles for reproductive improvement. J Fertil. 2015;3(145):17-9.

30. Durfey CL, Burnett DD, Liao SF, Steadman CS, Crenshaw MA, Clemente HJ, et al. Nanotechnology-based selection of boar spermatozoa: growth development and health assessments of produced offspring. Livest Sci. 2017;205(Supplement C):137-42.

31. Odhiambo JF, DeJarnette JM, Geary TW, Kennedy CE, Suarez SS, Sutovsky M, et al. Increased conception rates in beef cattle inseminated with nanopurified bull semen. Biol Reprod. 2014;91(4):97.

32. Lee WY, Lee R, Kim HC, Lee KH, Cui XS, Kim NH, et al. Pig spermatozoa defect in acrosome formation caused poor motion parameters and fertilization failure through artificial insemination and in vitro fertilization. Asian Australas J Anim Sci. 2014;27(10):1417.

33. Niżański W, Partyka A, Prochowska S. Evaluation of spermatozoal function—useful tools or just science. Reprod Domest Anim. 2016;51(S1):37-45.

34. Knox RV. Semen processing, Extending \& storage for artificial insemination in swine. Swine Reproductive Extension Specialist Department of Animal Sciences, University of Illinois, USA. 2011.

35. Vasquez ES, Feugang JM, Willard ST, Ryan PL, Walters KB. Bioluminescent magnetic nanoparticles as potential imaging agents for mammalian spermatozoa. J Nanobiotechnol. 2016;14(1):20.

36. Feugang JM, Youngblood RC, Greene JM, Fahad AS, Monroe WA, Willard ST, et al. Application of quantum dot nanoparticles for potential non-invasive bio-imaging of mammalian spermatozoa. J Nanobiotechnol. 2012:10(1):45

37. Feugang JM, Rodriguez-Munoz JC, Dillard DS, Crenshaw MA, Willard ST, Ryan PL. Beneficial effects of relaxin on motility characteristics of stored boar spermatozoa. Reprod Biol Endocrinol. 2015;13(1):24.

38. Martinez-Alborcia MJ, Morrell JM, Parrilla I, Barranco I, Vazquez JM, Martinez EA, et al. Improvement of boar sperm cryosurvival by using single-layer colloid centrifugation prior freezing. Theriogenology. 2012;78(5):1117-25.

39. Lassalle B, Testart J. Lectins binding on human sperm surface increase membrane permeability and stimulate acrosomal exocytosis. Mol Hum Reprod. 1996;2(9):651-8.

40. Sutovsky P. Proteomic analysis of mammalian gametes and sperm-oocyte interactions. Control of Pig Reproduction VIII. 2010;66:103.

41. Wu W, Wu Z, Yu T, Jiang C, Kim W-S. Recent progress on magnetic iron oxide nanoparticles: synthesis, surface functional strategies and biomedical applications. Sci Technol Adv Mater. 2015;16(2):023501.

42. Bepari RA, Bharali P, Das BK. Controlled synthesis of a-and $y$-Fe 203 nanoparticles via thermolysis of PVA gels and studies on a-Fe 203 catalyzed styrene epoxidation. J Saudi Chem Soc. 2014

43. Falchi L, Khalil WA, Hassan M, Marei WFA. Perspectives of nanotechnology in male fertility and sperm function. Int J Vet Sci Med. 2018;6(2):265-9.

44. Vermes I, Haanen C, Steffens-Nakken H. Reutellingsperger C. a novel assay for apoptosis flow cytometric detection of phosphatidylserine expression on early apoptotic cells using fluorescein labelled annexin V. J Immunol Methods. 1995;184(1):39-51.

45. Nicolson GL, Usui N, Yanagimachi R, Yanagimachi H. Lectin-binding sites on the plasma membranes of rabbit spermatozoa: changes in surface receptors during epididymal maturation and after ejaculation. J Cell Biol. 1977;74(3):950-62.

46. Schwarz MA, Koehler JK. Alterations in lectin binding to Guinea pig spermatozoa accompanying in vitro capacitation and the acrosome reaction. Biol Reprod. 1979;21(5):1295-307.
47. Caldeira DF, Paulini F, Silva RC, Azevedo RBd, Lucci CM. In vitro exposure of bull sperm cells to DMSA-coated maghemite nanoparticles does not affect cell functionality or structure. Int J Hyperth 2017(just-accepted):1-26.

48. Singh N, Jenkins GS, Asadi R, Doak S. Potential toxicity of superparamagnetic iron oxide nanoparticles (SPION). Nano Rev. 2010;1(1):5358.

49. Gupta AK, Wells S. Surface-modified superparamagnetic nanoparticles for drug delivery: preparation, characterization, and cytotoxicity studies. IEEE Trans Nanobioscience. 2004;3(1):66-73.

50. Knox RV. Artificial insemination in pigs today. Theriogenology. 2016; 85(1):83-93.

51. Knox R, Levis D, Safranski T, Singleton W. An update on north American boar stud practices. Theriogenology. 2008;70(8):1202-8.

52. Katz DF, Diel L, Overstreet JW. Differences in the movement of morphologically normal and abnormal human seminal spermatozoa. Biol Reprod. 1982:26(4):566-70.

53. Katz DF, Drobnis EZ, Overstreet JW. Factors regulating mammalian sperm MigrationThrough the female ReproductiveTract and oocyte vestments. GameteRes. 1989;22:26.

54. Liu DY, Clarke GN, Baker HW. Relationship between sperm motility assessed with the Hamilton-thorn motility analyzer and fertilization rates in vitro. J Androl. 1991;12(4):231-9.

55. Kawaguchi T, Kawachi M, Morikawa M, Kazuta H, Shibata K, Ishida M, et al. Key parameters of sperm motion in relation to male fertility. J Toxicol Sci. 2004;29(3):217-31.

56. Yanagimachi R. Fertility of mammalian spermatozoa: its development and relativity. Zygote. 1994;2(4):371-2.

57. Hernandez M, Roca J, Calvete JJ, Sanz L, Muino-Blanco T, Cebrian-Perez JA, et al. Cryosurvival and in vitro fertilizing capacity Postthaw is improved when boar spermatozoa are frozen in the presence of seminal plasma from good freezer boars. J Androl. 2007;28(5):689-97.

58. Yeste M. Recent advances in boar sperm cryopreservation: state of the art and current perspectives. Reprod Domest Anim. 2015;50:71-9.

Ready to submit your research? Choose BMC and benefit from:

- fast, convenient online submission

- thorough peer review by experienced researchers in your field

- rapid publication on acceptance

- support for research data, including large and complex data types

- gold Open Access which fosters wider collaboration and increased citations

- maximum visibility for your research: over $100 \mathrm{M}$ website views per year

At $\mathrm{BMC}$, research is always in progress.

Learn more biomedcentral.com/submissions 